\title{
Reservoir permeability from seismic attribute analysis
}

Gennady Goloshubin, University of Houston, Texas, U.S.

Dmitry Silin, University of California at Berkeley, California, U.S.

Vjacheslav Vingalov, Gleb Takkand, and Monir Latfullin, Lukoil, Tyumen, Russia

In case of porous fluid-saturated medium the Biot's poroelasticity theory predicts a movement of the pore fluid relative to the skeleton on seismic wave propagation through the medium. This phenomenon opens an opportunity for investigation of the flow properties of the hydrocarbon-saturated reservoirs. It is well known that relative fluid movement becomes negligible at seismic frequencies if porous material is homogeneous and well cemented. In this case the theory predicts an underestimated seismic wave velocity dispersion and attenuation. Based on Biot's theory, Helle et al. (2003) have numerically demonstrated the substantial effects on both velocity and attenuation by heterogeneous permeability and saturation in the rocks. Besides fluid flow effect, the effects of scattering (Gurevich, et al., 1997) play very important role in case of finely layered porous rocks and heterogeneous fluid saturation. We have used both fluid flow and scattering effects to derive a frequency-dependent seismic attribute which is proportional to fluid mobility and applied it for analysis of reservoir permeability.

\section{Reservoir model}

The presence of heterogeneities like high permeable channels has a significant impact on the flow properties of reservoir rock. Two or more scales of permeability are usually observed. Recent studies suggest that even in a "classical" porous rock, such as sandstone, the fluid flows through a very small portion of the pore space, while the most part of it is in stagnation. A connected system of highly permeable channels, due to relatively simple geometry of the pore space, provides the reservoir fluid flow. The rest part of the reservoir, due to the tortuous pores and pore throats, is significantly less permeable. At the same time, the total volume of highly permeable channels is usually small relatively to whole reservoir volume which contains most of the reservoir fluid. This contrast leads to the dual medium model of reservoir rock, which was originally proposed by Barenblatt et al. (1960). According to this model, the fluid flow in matrix 
blocks is local. It only supports the local exchange of fluid between individual blocks and the surrounding high permeable channels. In a large scale, fluid flows through the high permeable channels only. A combination of the Barenblatt's approach with Biot's theory of elastic wave propagation in permeable porous media leads to a model, which we call Biot-Barenblatt poroelastic model. In such model a compression P-wave is a superposition of slow and fast waves. For a permeable boundary one has to take into account two coupled incident waves, fast and slow. Each of the two coupled incident waves, fast and slow, generates a pair of fast and slow reflected and transmitted waves. Although the waves are coupled the superposition principle allows for considering each incident wave separately. We denote the respective transmission and reflection coefficients by $\mathrm{T}_{\mathrm{FF}}, \mathrm{T}_{\mathrm{FS}}, \mathrm{R}_{\mathrm{FF}}, \mathrm{R}_{\mathrm{FS}}$, etc. The first and second subscripts denote the type of the incident and transmitted (or reflected) waves respectively. For example, $\mathrm{R}_{\mathrm{FS}}$ denotes the reflection coefficient corresponding to fast incident and slow reflected wave. We can write the reflection and transmission coefficients for the fast incident wave in an asymptotic form (Goloshubin \& Silin, 2006):

$R_{F F}=R_{F F}^{0}+R_{F F}^{1} \sqrt{\varepsilon}+O(|\varepsilon|) ; T_{F F}=T_{F F}^{0}+T_{F F}^{1} \sqrt{\varepsilon}+O(|\varepsilon|) ; R_{F S}=R_{F S}^{1} \sqrt{\varepsilon}+O(|\varepsilon|) ; T_{F S}=T_{F S}^{1} \sqrt{\varepsilon}+O(|\varepsilon|)$. Where $R_{F F}^{0}=\frac{Z_{1}^{F}-Z_{2}^{F}}{Z_{1}^{F}+Z_{2}^{F}}$ and $T_{F F}^{0}=\frac{2 Z_{1}^{F}}{Z_{1}^{F}+Z_{2}^{F}}$ are zero order terms, were $\mathrm{Z}_{1}^{\mathrm{F}}$ and $\mathrm{Z}_{2}^{\mathrm{F}}$ are the impedances of the P-wave above and below the boundary. The first order terms content $T_{F F}^{1}$ coefficients, $R_{F F}^{1}$ coefficients, and parameter $\varepsilon$. The coefficients $T_{F F}^{1}$ and $R_{F F}^{1}$ depend on the ratio characterizing the contrast between the rock compressibility and fluid mobility in different parts of the reservoir. And $\varepsilon=i \frac{\rho_{f} \kappa \omega}{\eta}$ is a small dimensionless parameter, where $\rho_{f}$ is the density of pore fluid, $\kappa$ is reservoir rock permeability, $\eta$ is fluid viscosity, $\omega$ is the angular frequency of the signal and $i$ is the imaginary unity.

The reflection and transmission coefficients above suggest that the amplitude of the slow wave generated by the incident fast wave is small of the order of $\sqrt{\varepsilon}$ relative to the fast wave because it contents first order term only. The slow wave attenuates strongly. Therefore, in a thick layer, its contribution to the reflected signal is exclusively small. However, if the layer under consideration is very thin $(h<<\lambda)$, the slow wave can reach 
the other boundary and generate two pairs of slow and fast reflected and transmitted waves. Asymptotic analysis of the transformations slow-wave-to-slow-wave and slowwave-to-fast-wave suggests that the transmission and reflection coefficients include both frequency-independent zero order terms and frequency-dependent first order term:

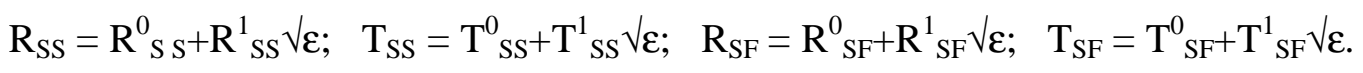

It means that in case of very thin porous permeable layer we need to take into account slow wave phenomenon. The response of a very thin permeable layer is a low-frequency resonance of the first order (Fig. 1) due to the slow waves.

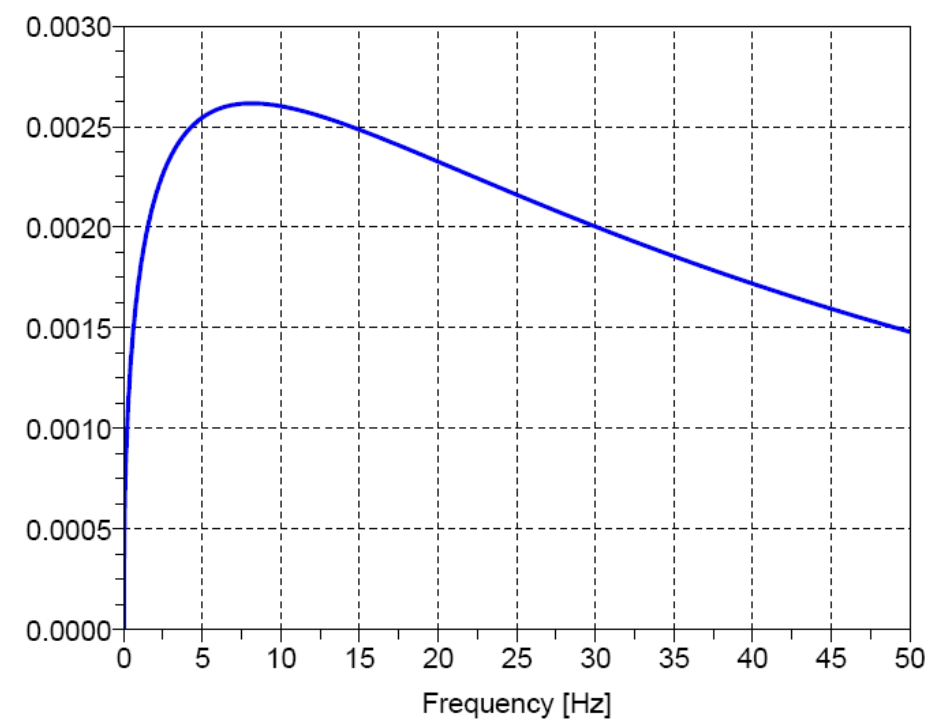

Fig. 1. Amplitude resonance of the first order at $8 \mathrm{~Hz}$ due to the slow waves: thickness of the permeable layer is $0.5 \mathrm{~m}$, permeability $k=1 \mathrm{D}$, and viscosity $\eta=0.003 \mathrm{~Pa}$-s.

A complete account for the impact of the layered structure of the reservoir on the reflection and transmission coefficients requires superposition of the formulas obtained here. In particular, for the reservoir model (Fig.2) we need to calculate the reflection coefficient from the reservoir top, reflection coefficients from the permeable lenses, and reflection coefficient from the reservoir bottom taking into account both transmission and absorption losses. 

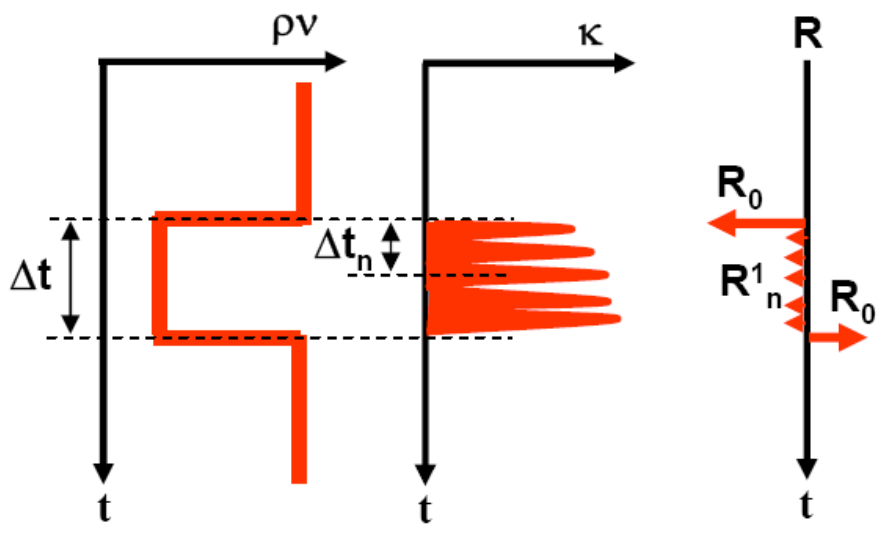

Fig.2. Reservoir model of a porous layer (left) with high-permeability lenses (middle) and the model response on incident fast $\mathrm{P}$-wave (right): $\mathrm{R}^{0}$ are zero order amplitudes of the reflections from reservoir top and bottom; $\mathrm{R}_{\mathrm{n}}^{1}$ are first order response from the lens $n ; \Delta t$ is two way time thickness of the reservoir, and $\Delta t_{\mathrm{n}}$ is time delay of seismic response from lens $n$

In this case the reflection coefficient from the inhomogeneous reservoir with number $(\mathrm{N})$ permeable lenses can be written in the form:

$$
\left.R(\omega) \approx R_{F F}^{0}-\left[1-\left(R_{F F}^{0}\right)^{2}\right] R_{F F}^{0} e^{-i \omega \Delta t-\alpha(\omega)}+\sum_{n=1}^{N} R_{n}^{1} \sqrt{(i} \omega \rho_{f} k_{n} / \eta\right) e^{-i \omega \Delta t_{n}-\alpha_{n}(\omega)}
$$

where $\omega=2 \pi \mathrm{f}$ is angular frequency, $\Delta t / 2$ is time thickness of the reservoir, $\alpha(\omega)$ describes both transmission energy loses and absorption for reflected fast P-wave from the bottom, $\Delta \mathrm{t}_{\mathrm{n}}$ and $\alpha_{\mathrm{n}}(\omega)$ are time delay and transmission-absorption loses for seismic response from lens $n, \mathrm{R}_{\mathrm{FF}}^{0}$ is reflection coefficient of the zero order from reservoir surface, and $\mathrm{R}_{\mathrm{n}}^{1}$ is reflection coefficient of the first order from lens $n$. The functional structure of the result has a similar asymptotic form as the formula for reflection coefficient of the fast P-wave in case of single permeable boundary. It includes both zero order and first order terms. The figure below shows a behavior of the fluid-saturated reservoir reflectivity as a function of frequency for a number of high permeable lenses (Fig.3). 


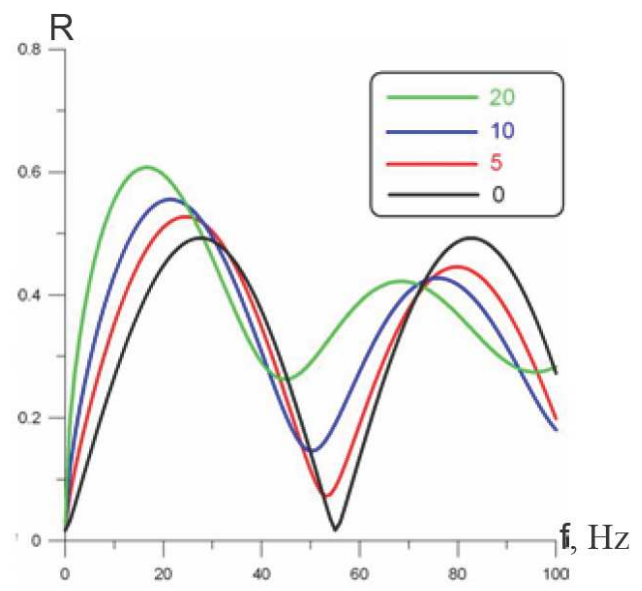

Fig.3. Reflection coefficient from a fluid-saturated inhomogeneous reservoir with different number $(0,5,10,20)$ of permeable lenses.

We can see the influence of the permeable lenses on reservoir reflectivity. There is perceptible shift of the first resonance frequencies to low frequency domain if the number of high permeable lenses is increased. It demonstrates a possibility for the investigation of the reservoir transport properties.

\section{Attribute analysis}

The model (Eq.1) above suggests seismic attributes which depend on the reservoir fluid mobility (permeability). In particular, for sediments with low impedance contrast and little lateral variation, the seismic amplitude response from reservoir at fixed low seismic frequency can be presented in a form:

$$
\left.R(\omega)_{\omega=\omega_{L}} \approx a+\sqrt{i} \sum_{n=1}^{N} b_{n} \sqrt{(} \rho_{f} k_{n} / \eta\right)
$$

Where $\alpha$ and $b_{n}$ are some constants. The constant $\alpha$ reflects an impedance contrast on reservoir surface and $b_{n}$ defines a wait in mechanism of fluid mobility averaging. In case of homogeneous fluid saturation, the amplitude response (Eq. 2) becomes proportional to average reservoir permeability. Additional transformations produce a base for calculation of the attribute which is proportional to weighted reservoir permeability.

$$
\left[R\left(\omega_{L}\right)-a\right]^{2} e^{-\pi / 2} \approx \frac{\rho_{f}}{\eta}\left(\sum_{n=1}^{N} b_{n} \sqrt{k_{n}}\right)^{2}
$$


To get this attribute we need to choose narrow frequency band wavelet at low seismic frequency and do wavelet transform of the reflection from reservoir zone, and also provide the additional transformations in accordance with formula (3). In this case the constant $\alpha$ plays role of amplitude discriminator and exponential term prescribes phase shift.

We have investigated the permeability influence on seismic reservoir response based on well and 3D seismic data from Kogalym oil field, Western Siberia. The structural map of the top of the reservoir with well locations is presented in Fig. 4. Mapping of the reservoir depth is based on both well and 3D seismic data.

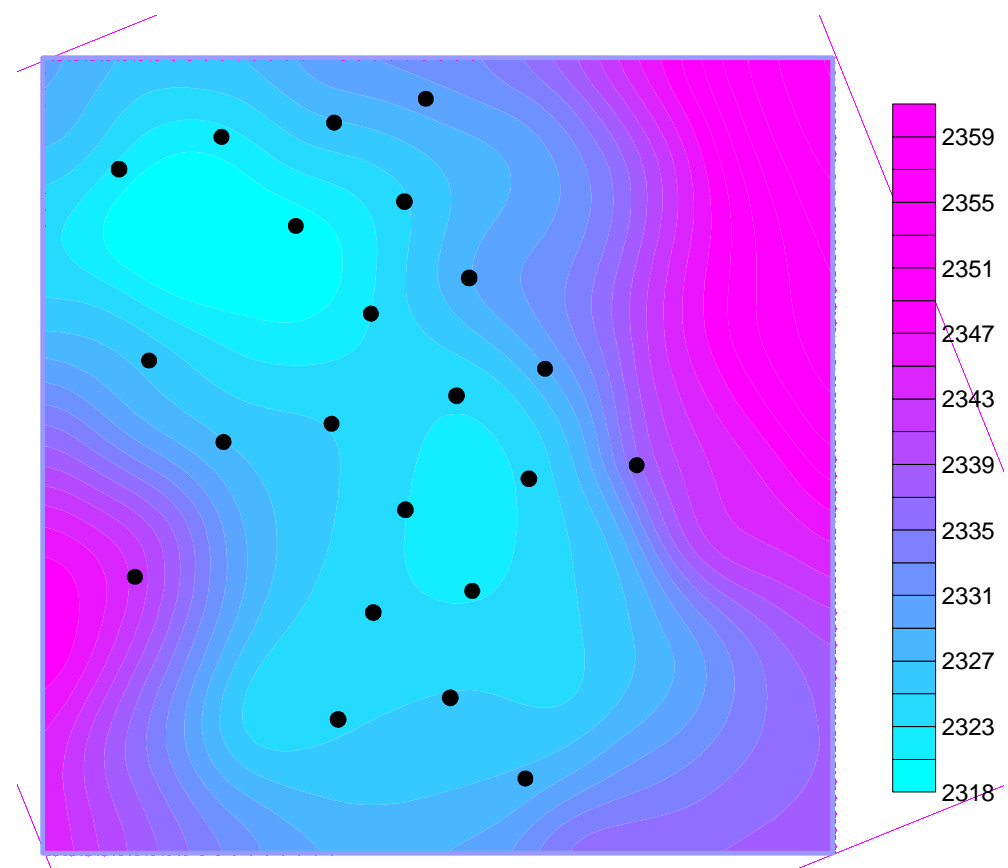

Fig.4. Structural map of the top of the reservoir with well locations (black points). Color bar shows the depth in meters.

In general the reservoir is inhomogeneous. We have used a probability $P i$ of occurrence of a permeable lens within reservoir to characterize reservoir heterogeneity. In our case, $P i$ is equal to the ratio of cumulative effective thicknesses of the permeable lenses to the total thickness of the reservoir: $\mathrm{P}_{\mathrm{i}}=\Sigma \mathrm{H}_{\text {eff }} / \mathrm{H}_{\text {total }}$. The analysis of the well data shows that the weighted average permeability can be separated for two gradations within reservoir at least by probability Pi (Fig. 5). First group of the data is concentrated within $\mathrm{Pi}=0.1-0.3$. And second group has $\mathrm{Pi}=0.3-0.6$. 


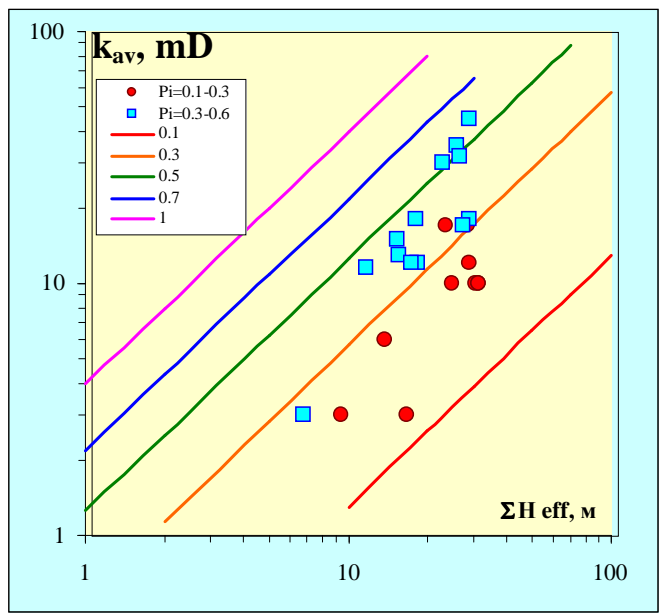

Fig.5 Average reservoir permeability vs. cumulative effective thickness of the reservoir can be separated for two gradations: $\mathrm{Pi}=0.1-0.3$ and $\mathrm{Pi}=0.3-0.6$

Seismic attribute calculation was performed using wavelet transform and its transformations according to the formula (3). Figure 6 shows the map of seismic attribute along the reservoir surface.

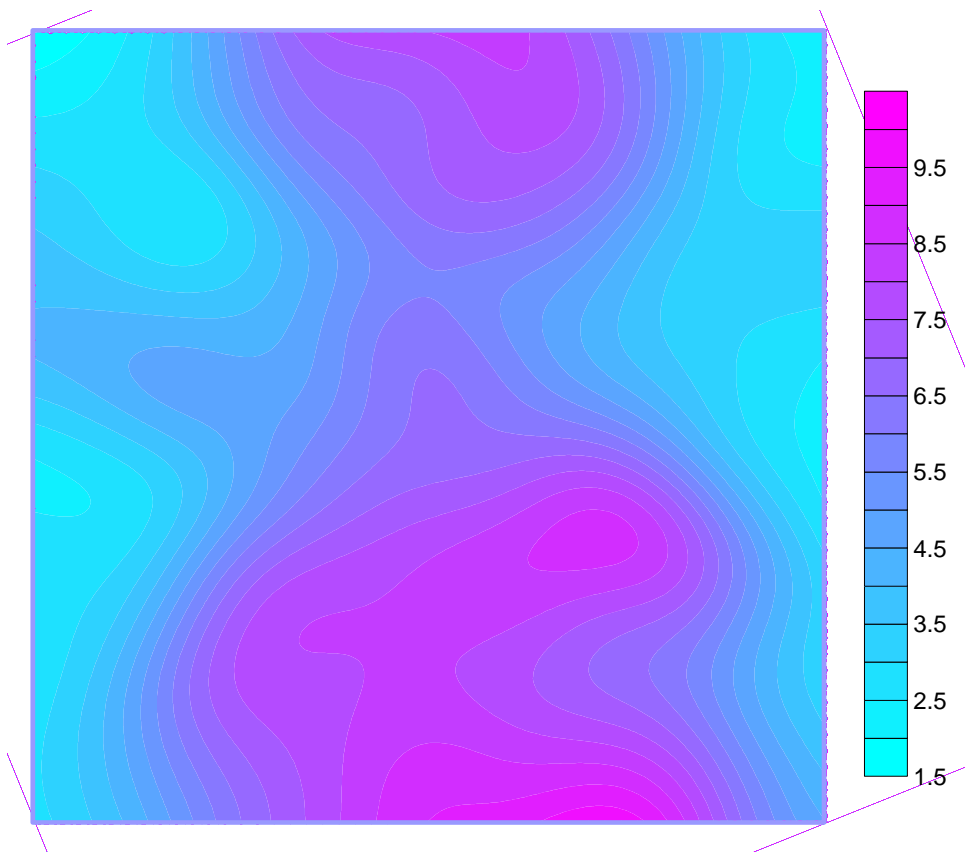

Fig.6. Seismic attribute map along reservoir surface

Note that the structural map (Fig. 4) is not in agreement with the attribute map (Fig. 6). Thus, the structure only does not control the attribute behavior. A comparison of the 
average reservoir permeability with seismic attribute shows an influence of the permeability to seismic data (Fig. 7). If the probability $P i$ of occurrence of a permeable lens within reservoir is order of 0.1-0.3 (first group gradation) there is no possibility for reliable permeability prognosis. There is a tight connection between seismic attribute and average permeability within second group gradation $(\mathrm{Pi}=0.3-0.6)$. In this case the reliability of a permeability prognosis is equal to 0.9 .

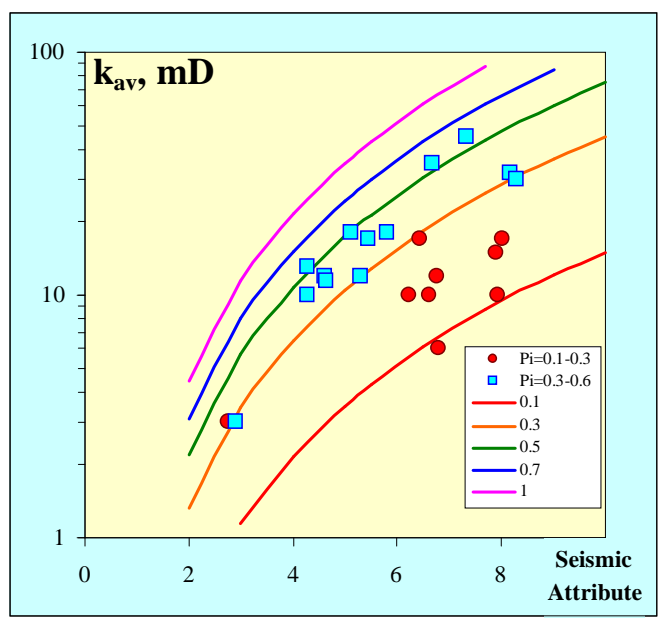

Fig.6 Average reservoir permeability vs. seismic attribute for different Pi.

Based on a poroelasticity model of wave propagation and $\log$ and seismic data analysis we suggest seismic imaging of the reservoir transport properties, in particular lateral permeability variations, is realistic. Vertical reservoir heterogeneity plays important role. Taking into account the influence of this type heterogeneity may help to achieve the best result for mapping of lateral variations of the reservoir permeability based on seismic and log data.

\section{Suggested reading:}

"Basic concepts in the theory of seepage of homogeneous liquids in fissured rocks" by Barenblatt et al. (Journal of Applied Mathematics, v. 24, 1960). "Velocity and attenuation in partially saturated rocks: poroelastic numerical experiments" by Helle et al. (Geophysical Prospecting, 51, 2003). "Seismic attenuation in finely layered porous rocks: Effects of fluid flow and scattering" by Gurevich et al. (Geophysics, v. 62, No 1, 1997). "Frequency-dependent seismic reflection from a permeable boundary in a fractured reservoir" by Goloshubin \& Silin (SEG Expanded Abstracts, 2006). 